DOT:10.1145/1516046.1516047

\title{
ACM-W Celebrates Women in Computing
}

\section{Computer science is no longer the hot, high-enrollment field it once was.}

This is not news. While many suggestions have been made for increasing enrollments, it is unlikely that computer science will ever be as vibrant as it could be-and should be-as long as a large portion of the talent pool remains underrepresented. After all, if we are missing the best and the brightest of a group who can offer exciting ideas that would enrich the field, computer science suffers. In addition, different groups often present different perspectives - a scenario completely lost when we do not encourage diversity.

With this in mind, the mission of the ACM Women's Council (ACM-W) is to inform and support women in computing. Since ACM is an international organization, this means developing programs with a worldwide reach; with something for each of ACM's very broad constituencies: K-12 students, undergraduates at liberal arts and research institutions, master's and Ph.D. students, faculty from all types of institutions, and women in industry and government working as computer practitioners and researchers. Increasingly, we strive to partner both with other segments within ACM and other organizations dedicated to improving gender diversity.

Some of our active programs include scholarships to help women students attend research conferences. This effort is not aimed at the advanced Ph.D. student who has already committed to a career in academia or industrial research. Rather we look to support the undergraduate woman by giving her a chance to see the types of options available and encourage her to continue on to graduate school. Similarly we hope to encourage the master's student to aim for a Ph.D. We offer up to $20 \$ 500$ scholarships per year. Moreover, we have recently asked the ACM's Special Interest Groups (SIGs) to partner with us by offering scholar ship recipients complimentary regis tration as well as provide conference mentors to help them learn the ropes. We are thrilled by the response we have received from many of the SIGs.

Another program involving SIG cooperation is our Athena Lecturer Award honoring the most outstanding women scholars. It was established to address the fact that women are often overlooked when nominations are considered for advanced membership grades or awards. The goal of the Athena Lecturer Award is to celebrate women's scholarship and technical contributions to the field as well as increase the visibility of women scholars. Rather than asking for individual nominations, each SIG is invited to nominate their most outstanding women scholars. By using this format, we encourage SIGs to think about promoting women in the field, and hopefully remember these women when they are nominating people for other awards or selecting keynote speakers or program chairs for future conferences.

Many readers will be familiar with the Grace Hopper Celebration of Women. To keep the Hopper momentum going throughout the year, ACMW offers regional Hopper-like events designed to attract attendees within a two-hour driving radius of each other.
Elaine Wevuker

Not only does this make it relatively inexpensive to attend meetings since students and faculty often travel together, the proximity also helps establish and maintain a local community of women pursuing a common goal. We have sponsored quite a number of these meetings both within the U.S. and Australia, with one being planned in Turkey.

Another unique ACM-W initiative is the Ambassador program in which a woman serves as the Ambassador from her country and shares information about the climate there for women in computing. At times we have had representatives from six different continents. We are now developing our first internationally distributed program aimed at attracting middle school girls to computer science by adapting a successful program to several different cultures.

This is just a sampling of the many programs within ACM-W created to promote and further advance women in the computing field. Readers are encouraged to visit our Web site at http:// women.acm.org to learn about the full range of programs and initiatives offered. ACM-W is an all-volunteer organization open to anyone interested in improving gender diversity. If you see a project that interests you, please consider volunteering. If you have an idea for a new project, let us know. Take a look at our newsletter to see project details, read interviews with outstanding women, and learn about upcoming events.

Diversity is not the problem of the underrepresented group. It is everyone's problem. If we want our field to grow and flourish, we need the contribution of talented people of all types. $\mathbf{C}$

Elaine Weyuker is chair of ACM-W and is a researcher at AT\&T Labs specializing in empirical software engineering and testing research. 